\title{
ASSOCIAÇÃO ENTRE INDICADORES DE PRÁTICA DE ATIVIDADES FÍSICAS NA ADOLESCÊNCIA COM O NÍVEL ATUAL DE PRÁTICA DE ATIVIDADES FÍSICAS NO LAZER EM ACADÊMICOS DE UM CURSO DE EDUCAÇÃO FÍSICA NO NORDESTE DO BRASIL
}

\author{
Thiago Ferreira de Sousa \\ Universidade Federal de Santa Catarina, Florianópolis, Santa Catarina, Brasil.
}

\section{Sueyla Ferreira da Silva dos Santos}

Universidade Estadual do Sudoeste da Bahia, Jequié, Bahia, Brasil.

Ana Clara Souza Pie

Escola Nacional de Saúde Pública Sergio Arouca, Rio de Janeiro, Brasil.

Luana Callegaro Rossato

Universidade Federal de Santa Catarina, Florianópolis, Santa Catarina, Brasil.

\begin{abstract}
Resumo
O objetivo deste estudo foi identificar a frequência de inativos no lazer atual e sua associação com a prática de atividade física na adolescência, no contexto escolar e não escolar em acadêmicos de um curso de Educação Física. Foi realizado um estudo transversal com 105 estudantes do curso. A frequência de acadêmicos inativos no lazer foi $23,1 \%$, com diferença significativa entre os gêneros ( $p=0,003)$ e maior frequência entre as mulheres (38,6\%). Associações estatísticas foram evidenciadas em acadêmicos inativos hoje quando perguntados sobre a prática realizada na adolescência no contexto não escolar $(\mathrm{p}=0,047)$. Desse modo, vê-se a importância de o professor de Educação Física estimular à prática de atividades físicas desde as idades iniciais, visando à adesão à prática ao longo da vida.

Palavras-Chave: Lazer - Adolescente - Estudantes.
\end{abstract}

\section{Introdução}

A inatividade física representa um problema atual para a saúde pública (WHO, 2002), tendo em vista sua relação com maiores chances de mortalidade por problemas cardiovasculares (PAFFENBARGER et al., 1986; PAFFENBARGER et al., 1993; YUSUF et al., 2004), assim como por outras morbidades (WHO, 2002).

A adoção de um estilo de vida ativo é muito importante para uma vida duradoura e com qualidade, principalmente pelos potenciais benefícios amplamente relatados na literatura, provenientes de sua prática sistemática (UNITED STATES DEPARTMENT OF HEALTH AND HUMAN SERVICES - USDHHS, 1996). Desse modo, divulgar e ampliar o alcance das recomendações mínimas para a prática de atividades físicas na população de adultos torna-se essencial para adoção e manutenção de comportamentos saudáveis (UNITED STATES DEPARTMENT OF HEALTH AND HUMAN SERVICES - USDHHS, 2008).

Em estudos com subgrupos populacionais, como os universitários, constata-se uma abordagem pautada em diferentes características do estilo de vida (GIROTTO et al., 1996; RABELO et al., 1999; OLIVEIRA et al., 2007; ABOLFOTOUH et al., 2007; FRANCA; COLARES, 2008), em especial relativo à atividade física, englobando os quatro domínios (GUEDES; SANTOS; LOPES, 2006; SILVA et al., 
2007) ou somente o lazer (STOCK et al., 2001; ABOLFOTOUH et al., 2007). Em contrapartida, as informações provenientes do nível de atividade física pregressa, ou seja, atividades físicas realizadas em idades anteriores à fase adulta (infanto-juvenis), não são bem documentadas (ALVES et al., 2005), principalmente em populações específicas da Região Nordeste do Brasil.

A literatura demonstra que há relação positiva entre o nível de atividade física na adolescência e o nível de atividade física na fase adulta (TAMMELIN et al., 2003; TELAMA et al., 2005). Tendo em vista essas observações, existe relação entre a prática de atividade física no lazer atual e a prática de atividade física realizada na adolescência em um ambiente escolar e não escolar em universitários?

O objetivo deste estudo foi identificar a frequência de inativos no lazer atual e sua associação com a prática de atividade física na adolescência, no contexto escolar e não escolar, em acadêmicos do curso de Educação Física de uma instituição pública do Nordeste do Brasil.

\section{Métodos}

A presente pesquisa é derivada do estudo de característica transversal Perfil dos Indicadores da Aptidão Física e Saúde dos Estudantes de Educação Física da Universidade Estadual de Santa Cruz (PAFIS - UESC/BAHIA), realizado em uma universidade pública do Estado da Bahia, Região Nordeste do Brasil. Informações sobre o estudo PAFIS - UESC/BAHIA foram apresentadas anteriormente (SOUSA, 2009).

A coleta foi realizada no mês de maio de 2007 e todos os acadêmicos do curso de Licenciatura em Educação Física foram convidados a participar da pesquisa, sendo elegíveis somente os estudantes que estavam matriculados e desempenhando suas atividades frequentemente $(\mathrm{N}=143)$. A coleta de dados foi realizada em salas de aulas do curso, durante três semanas, sendo assim, os estudantes que estavam presentes nas salas foram informados sobre os objetivos e a forma de participação no estudo.

Para a realização do estudo, foi feito um treinamento prévio com os pesquisadores responsáveis para a aplicação dos testes de aptidão física e dos questionários. $\mathrm{O}$ instrumento de coleta foi preenchido pelos estudantes de forma livre, todavia as dúvidas foram esclarecidas pelos pesquisadores responsáveis pela coleta que assistiram os estudantes durante o preenchimento.

O instrumento foi construído tendo como base questionários previamente validados em estudos populacionais (BARROS, 1999; BRASIL, 2004) e composto pelas seguintes seções: informações sóciodemográficas, indicador de saúde e qualidade de vida, estilo de vida (atividade física, hábitos alimentares, controle do estresse, comportamentos preventivos e relacionamentos), satisfação e controle da massa corporal.

Para o presente estudo, as variáveis foram categorizadas conforme demonstrado no Quadro 1. 


\begin{tabular}{|l|l|}
\hline Variável & Critérios de categorização \\
\hline Sociodemográficas & $\begin{array}{l}\text { Faixa etária: até } 22 \text { anos e } 23 \text { anos ou mais; } \\
\text { Turma: referente ao ano de ingresso no curso (2004, 2005, } \\
\text { 2006 e 2007); } \\
\text { Renda familiar bruta: múltiplos do salário mínimo, que na época } \\
\text { era R\$ 350,00 (até R\$1.750,00 e R\$ R\$ 1.751,00 ou mais). }\end{array}$ \\
\hline Atividade física escolar & $\begin{array}{l}\text { Inativo - afirmar raramente ou nunca ter frequentado as aulas } \\
\text { de Educação Física, mesmo tendo oportunidade de participar; } \\
\text { Ativo - referir ter participado das aulas de Educação Física } \\
\text { no Ensino Fundamental e/ou Médio. }\end{array}$ \\
\hline Atividade física não escolar & $\begin{array}{l}\text { Inativo - afirmar nunca ter frequentado escolinhas ou equipes } \\
\text { de esporte, danças ou lutas; } \\
\text { Ativo - referir ter participado de escolinhas ou equipes de } \\
\text { esporte, danças ou lutas em forma de competição e/ou apre- } \\
\text { sentação. }\end{array}$ \\
\hline $\begin{array}{l}\text { Inativo - referir não ter praticado atividade física no lazer nas } \\
\text { duas semanas anteriores a coleta de dados, como: exercícios } \\
\text { físicos (ginástica, caminhada, corrida), esportes, danças ou artes } \\
\text { marciais; } \\
\text { Ativo - afirmar a prática de atividade física no mínimo uma } \\
\text { vez por semana. }\end{array}$ \\
\hline Atividade física atual
\end{tabular}

Quadro 1 - Variáveis e critérios de categorização.

Os participantes do estudo PAFIS - UESC/BAHIA foram informados acerca do anonimato e da participação voluntária na pesquisa, assim como a possibilidade de retirada do consentimento a qualquer ocasião. Essas informações foram fornecidas anteriormente à aplicação do instrumento de pesquisa que ocorreu nas salas do curso. As condições de aplicação foram semelhantes para as quatro turmas e a coleta de dados somente iniciou após a assinatura do Termo de Consentimento Livre e Esclarecido (TCLE), conforme um dos procedimentos constantes na Resolução 196/96.

Os dados foram analisados no pacote estatístico SPSS versão 16.0, visando à aplicação de procedimentos de estatística descritiva, como média e frequência. Posteriormente foram 2) para 2 ) e Qui-quadrado ( $\chi$ utilizados os testes de Qui-quadrado ( tendência (quando conveniente) e teste Fisher's Exact, considerando o valor de significância de $\mathrm{p}<0,05$.

\section{Resultados}

Informações acerca das características sóciodemográficas dos estudantes são apresentadas na Tabela 1. Dos 143 estudantes regularmente matriculados no curso, participaram da presente pesquisa 105 acadêmicos, o que equivale a uma perda de aproximadamente $27,0 \%$. Tal perda ocorreu pela ausência dos alunos em sala de aula durante o período da coleta, sendo que nenhum sujeito recusou participar do estudo. A idade média dos estudantes foi de 23,2 anos $( \pm 4,9 ; 17$ a 42$)$ e as médias de idade dos homens foi de 23,5 anos $( \pm 5,4 ; 17$ a 42$)$ e das mulheres de $22,7( \pm 4,2 ; 18$ a 35$)$. A maior frequência de indivíduos participantes foi do gênero masculino $(57,1 \%)$ e da turma referente 
ao ano de 2004 (28,6\%). Perdas de dados para a variável renda familiar bruta foram evidenciadas e tais informações são referentes a apenas 94 acadêmicos do curso.

Tabela 1. Informações sóciodemográficas dos estudantes, por gênero.

\begin{tabular}{|c|c|c|c|c|}
\hline \multirow{2}{*}{ Variáveis } & Homens & Mulheres & Total & \\
\hline & $\mathbf{\%}(\mathbf{N})$ & $\mathbf{\%}(\mathbf{N})$ & $\mathbf{\%}(\mathbf{N})$ & $\mathbf{p}$ \\
\hline Faixa etária & & & & $0,923 \#$ \\
\hline Até 22 anos & $53,6(30)$ & $54,5(24)$ & $54,0(54)$ & \\
\hline 23 anos ou mais & $43,4(26)$ & $45,5(20)$ & $46,0(46)$ & \\
\hline Turma & & & & $0,052 \# \#$ \\
\hline 2004 & $23,3(14)$ & $35,6(16)$ & $28,6(30)$ & \\
\hline 2005 & $21,7(13)$ & $26,7(12)$ & $23,8(25)$ & \\
\hline 2006 & $28,3(17)$ & $24,4(11)$ & $26,7(28)$ & \\
\hline 2007 & $26,7(16)$ & $13,3(06)$ & $21,0(22)$ & \\
\hline Renda & & & & $0,162 \#$ \\
\hline Até R\$ 1.750,00 & $59,3(32)$ & $42,5(17)$ & $52,1(49)$ & \\
\hline R\$ 1.751,00 ou mais & $40,7(22)$ & $57,5(23)$ & $47,9(45)$ & \\
\hline \# Teste Qui-quadrado (c2); \#\# Teste Qui-quadrado(c2) para tendência; \#\#\# Teste Fisher”s Exact
\end{tabular}

A frequência de acadêmicos de Educação Física que afirmaram não terem realizado atividades físicas em nenhum dia nas duas semanas anteriores à coleta de dados foi de $23,1 \%$. As mulheres referiram não terem praticado atividades físicas no lazer com maior proporção que os homens, 38,6\% e 11,7\% respectivamente, e tal diferença foi estatisticamente significativa (Tabela 2).

Tabela 2. Frequência de Inatividade Física no Lazer (IFL) por variáveis sóciodemográficas.

\begin{tabular}{|c|c|c|}
\hline Variáveis & $\% \operatorname{IFL}(\mathrm{N})$ & $\mathbf{p}$ \\
\hline Gênero & & 0,003\# \\
\hline Masculino & $11,7(07)$ & \\
\hline Feminino & $38,6(17)$ & \\
\hline Faixa etária & & $0,222 \#$ \\
\hline Até 22 anos & $18,5(10)$ & \\
\hline 23 anos ou mais & $31,1(14)$ & \\
\hline Turma & & $0,164 \# \#$ \\
\hline 2004 & $30,0(09)$ & \\
\hline 2005 & $25,0(06)$ & \\
\hline 2006 & $21,4(06)$ & \\
\hline 2007 & $13,6(03)$ & \\
\hline Renda & & $0,371 \#$ \\
\hline Até $\mathrm{R} \$ 1.750,00$ & $20,8(10)$ & \\
\hline Acima de $\mathrm{R} \$ 1.751,00$ & $31,1(14)$ & \\
\hline \multicolumn{3}{|c|}{ \# Teste Qui-quadrado (c2); \#\# Teste Qui-quadrado (c2) para tendência \#\#\# Teste Fisher's Exact } \\
\hline
\end{tabular}


Apesar da associação entre inatividade física e gênero ter sido constatada nas análises considerando todos os sujeitos envolvidos na pesquisa, tal evidência não foi constatada nas análises estratificadas por gênero (Tabela 3). No entanto, frequências superiores de inatividade física foram demonstradas para o gênero feminino com destaque para as mulheres de idade igual ou superior a 23 anos (47,4\%), turma de início em 2006 (45,5\%) e faixa de renda familiar bruta acima de $\mathrm{R} \$ 1.751,00$ (47,8\%).

Tabela 3. Frequência de Inatividade Física no Lazer (IFL) por variáveis sóciodemográficas, por gênero.

\begin{tabular}{|c|c|c|c|c|}
\hline \multirow[t]{2}{*}{ Variáveis } & \multicolumn{2}{|c|}{ Homens } & \multicolumn{2}{|c|}{ Mulheres } \\
\hline & $\% \operatorname{IFL}(\mathrm{N})$ & $\mathbf{p}$ & $\%$ IFL (N) & p \\
\hline Faixa etária & & $0,231 \# \# \#$ & & $0,535 \#$ \\
\hline Até 22 anos & $6,7(02)$ & & $33,3(08)$ & \\
\hline 23 anos ou mais & $19,2(05)$ & & $47,4(09)$ & \\
\hline Turma & & 0,698\#\# & & $0,437 \# \#$ \\
\hline 2004 & $14,3(02)$ & & $43,8(07)$ & \\
\hline 2005 & $15,4(02)$ & & $36,4(04)$ & \\
\hline 2006 & $5,9(01)$ & & $45,5(05)$ & \\
\hline 2007 & $12,5(02)$ & & $16,7(01)$ & \\
\hline Renda & & 0,606\#\#\# & & $0,755 \#$ \\
\hline Até $\mathrm{R} \$ 1.750,00$ & $12,5(04)$ & & $37,5(06)$ & \\
\hline Acima de $\mathrm{R} \$ 1.750,00$ & $13,6(03)$ & & $47,8(11)$ & \\
\hline
\end{tabular}

Quanto à prática de atividade física na adolescência, considerando todo o grupo de acadêmicos, as análises demonstraram as seguintes frequências para o contexto não escolar: ativos $=80,8 \%$ e inativos $=19,2 \%$. Para o contexto escolar foram: ativos $=92,3 \%$ e inativos $=7,7 \%$. Associações significativas foram identificadas somente entre a atividade física praticada no lazer atualmente e a prática $2=3,948$ ) $\chi$ realizada no contexto não escolar ( $\mathrm{p}=0,047$; quando analisados em função dos acadêmicos de um modo geral. A frequência de inatividade física no lazer foi superior $(42,1 \%)$ para os acadêmicos que referiram inatividade física em âmbito não escolar (Tabela 4).

Tabela 4. Frequência de Inatividade Física no Lazer (IFL) por Atividade Física no Contexto Escolar e Não Escolar em estudantes do curso de Educação Física.

\begin{tabular}{|c|c|c|}
\hline Variáveis & IFL & p \\
\cline { 2 - 3 } & $\mathbf{\%}(\mathbf{N})$ & $0,382 \# \#$ \\
\hline Atividade Física Escolar & & \\
\hline Ativo & $21,9(21)$ \\
\hline Inativo & $37,5(03)$ \\
\hline Atividade Física Não Escolar & & $\mathbf{0 , 0 4 7 \#}$ \\
\hline Ativo & $17,9(15)$ \\
\hline Inativo & $42,1(08)$ \\
\hline \# Teste Qui-quadrado (c2); \#\# Teste Qui-quadrado (c2) para tendência; \#\#\# Teste Fisher's Exact \\
\hline
\end{tabular}


Em função do gênero (Tabela 5), a diferença estatística foi evidenciada somente para o gênero feminino $(\mathrm{p}=0,030)$ entre as mesmas variáveis que apresentaram associação na análise considerando todos os indivíduos. A proporção de mulheres inativas atualmente foi superior para a categoria referente à inatividade física no contexto não escolar (70,0\%) quando comparada às mulheres que afirmaram ter praticado $(29,4 \%)$.

Tabela 5. Frequência de Inatividade Física no Lazer (IFL) por Atividade Física no Contexto Escolar e Não Escolar em estudantes de Educação Física, por gênero.

\begin{tabular}{|c|c|c|c|c|}
\hline \multirow[t]{2}{*}{ Variáveis } & \multicolumn{2}{|c|}{ Homens } & \multicolumn{2}{|c|}{ Mulheres } \\
\hline & $\% \operatorname{IFL}(\mathrm{N})$ & $\mathbf{p}$ & $\% \operatorname{IFL}(\mathrm{N})$ & $\mathbf{p}$ \\
\hline Atividade Física Escolar & & 0,601\#\#\# & & $0,282 \# \# \#$ \\
\hline Ativo & $12,5(07)$ & & $35,0(14)$ & \\
\hline Inativo & $0(0)$ & & $75,0(03)$ & \\
\hline Atividade Física Não Escolar & & $0,647 \# \# \#$ & & 0,030\#\#\# \\
\hline Ativo & $10,0(05)$ & & $29,4(10)$ & \\
\hline Inativo & $11,1(01)$ & & $70,0(07)$ & \\
\hline
\end{tabular}

\section{Discussão}

O presente estudo buscou identificar a associação entre a prática de atividade física atual e a prática de atividade física pregressa, em dois contextos, escolar e não escolar. Tendo em vista a realização de um estudo do tipo transversal, destaca-se a impossibilidade de causalidade entre as informações observadas.

Além disso, apesar da limitação existente acerca do número reduzido de sujeitos nesta pesquisa $(\mathrm{N}=105)$, que inviabilizam análises controlando as variáveis de confundimento, o percentual de participantes $(73,0 \%)$ é representativo dos acadêmicos do curso de Educação Física da UESC. A não participação ocorreu, principalmente, pela ausência dos acadêmicos durante o período da coleta (três semanas).

Quanto à frequência de inatividade física atual encontrada $(23,1 \%)$, demonstrou ser inferior à prevalência de inatividade física evidenciada para a população do Brasil $(29,2 \%)$, bem como para a população da capital baiana, Salvador $(28,0 \%)$. Destaca-se que a prevalência citada se refere aos quatro domínios da atividade física (MOURA et al., 2008), diferentemente do presente estudo que somente identificou informações da prática de atividade física atual no contexto do lazer.

Quando comparado às informações disponíveis sobre universitários, constatase que, em relação ao estudo com acadêmicos de Educação Física da Universidade de Pelotas (BIELEMANN et al., 2007), a prevalência de inatividade física no lazer (prevalência geral: 25,8\%; escore inferior a 150 minutos/semana) foi superior nas mulheres $(35,3 \%)$ que nos homens $(15,2 \%)$, fato também evidenciado nos acadêmicos de Educação Física da UESC, sendo tais associações estatisticamente significativas em ambos os estudos $(\mathrm{p}=0,003)$. Outros estudos realizados com universitários também demonstraram a tendência evidenciada na presente pesquisa, quando analisados em função do nível de atividade física global (GUEDES; SANTOS; LOPES, 
2006; SILVA et al., 2007) e somente para o contexto do lazer (STOCK et al., 2001; ABOLFOTOUH et al., 2007). Estudos de abrangência populacional convergem com as informações sobre o nível de atividade física no lazer em função do gênero observado neste estudo (BARROS; NAHAS, 2001; PITANGA; LESSA, 2008; MOURA et al., 2008).

Diferentemente da prevalência de inatividade física atual identificada, outros estudos realizados com universitários demonstraram valores superiores em relação à inatividade física, como o estudo que englobou acadêmicos da área de saúde da Universidade de Brasília (Medicina, Nutrição, Farmácia, Enfermagem, Odontologia e Educação Física) (MARCONDELLI et al., 2008). A prevalência de acadêmicos insuficientemente ativos (gasto energético acerca das atividades físicas realizadas no dia anterior à coleta de dados) foi 65,5\% em função de todos os cursos, em contrapartida, os acadêmicos de Educação Física apresentaram prevalência de apenas 6,5\% em relação aos demais cursos.

Em recente estudo realizado com universitários ( $\mathrm{n}=871$ ) de Tocantins, Região Norte do Brasil (RODRIGUES et al., 2008), constatou-se, por meio da aplicação do International Physical Activity Questionnaire (IPAQ) - versão curta, prevalência de estudantes considerados sedentários de somente 7,6\%, com maior proporção de sedentários para os indivíduos de idade igual ou superior a 25 anos, em relação aos de idade inferior (< 25 anos), 38,3\% e 26,2\%, respectivamente. Nesta pesquisa não foi identificada associação estatística entre a atividade física e a faixa etária, no entanto os homens e as mulheres apresentaram frequências de inatividade física elevada na faixa de idade superior, o que representa uma característica demonstrada em diversos estudos realizados com universitários (GUEDES; SANTOS; LOPES, 2006; BIELEMANN et al., 2007) e populações (BARROS; NAHAS, 2001; HALLAL et al., 2003).

Além disso, em estudo realizado com universitários da área de saúde de duas universidades públicas do Estado de Pernambuco, Região Nordeste do Brasil, verificouse que $65,1 \%$ e $57,2 \%$ de acadêmicos do primeiro e do último semestre, respectivamente, demonstraram valores percentuais para a inatividade física na semana anterior à coleta de dados (FRANCA; COLARES, 2008) superiores aos dos acadêmicos da UESC. Informações acerca do progresso dos acadêmicos no curso da UESC reveleram um crescimento da frequência de inatividade física com o avanço dos anos no curso, principalmente para o gênero feminino, embora tais evidências não tenham apresentado associação linear. $\mathrm{O}$ crescimento da frequência de inatividade física no lazer nos acadêmicos pode estar atrelado a uma maior demanda de tarefas relacionadas ao curso, ou pode estar relacionada à transição dos acadêmicos para o mercado de trabalho, por meio de estágios ou atividades remuneradas na área.

Entretanto, maiores comparações entre os estudos são limitadas, principalmente pelo uso de medidas diferentes para avaliar o nível de prática de atividade física em universitários, tendo em vista que cada instrumento empregado abrange um ou mais domínios da prática de atividade física, seja no lazer, deslocamento, atividades ocupacionais ou domésticas. Contudo, as tendências identificadas para os estudos realizados com universitários (GUEDES; SANTOS; LOPES, 2006; SILVA et al., 2007; STOCK et al., 2001; ABOLFOTOUH et al., 2007) são semelhantes aos de abrangência populacional (HALLAL et al., 2003; MOURA et al., 2008). 
Em relação aos indicadores de atividade física pregressa empregados nas análises, tanto no contexto escolar como no não escolar, identificam-se possíveis limitações quanto ao recordatório das atividades físicas realizadas, pois as medidas apenas representam indicadores da prática regular, e podem estar sendo superestimadas, haja vista que representam comportamentos saudáveis aceitos pela sociedade. Além disso, não permitem a discriminação quanto à intensidade das atividades praticadas, tendo em vista que são informações importantes para a caracterização do nível de atividade física. Em linhas gerais, mediante a análise desses dois indicadores de atividade física pregressa, constataram-se frequências de inatividade física inferior à inatividade física no lazer atualmente (Inatividade Física Escolar: 7,7\%; Inatividade Física não Escolar: 19,2\%).

Observou-se a associação estatística entre a atividade física no lazer e a prática de atividade física não escolar ( $\mathrm{p}=0,047)$, representando assim um indicador da relação entre essas duas variáveis. Tais pressupostos foram relatados em demais estudos que indicaram maiores níveis de prática de atividade física em adultos que praticaram atividade física quando crianças e/ou adolescentes em um contexto não escolar (TELAMA et al., 2005; TAMMELIN et al., 2003; ALVES et al., 2005), assim como refutados quanto à existência dessa relação (GORDON-LARSEN et al., 2004). Contudo, Conroy et al. (2005) demonstraram relação entre a prática de atividade física tanto no ambiente escolar como no não escolar e a prática de atividade física em níveis recomendados na fase adulta.

Embora haja divergência entre os estudos, considera-se de baixa a moderada a relação entre a prática de atividade física na adolescência e a prática na fase adulta, conforme identificado em estudo realizado por meio de acompanhamento dos indivíduos ao longo dos anos (longitudinal) (TELAMA et al., 2005). Apesar dessa relação de baixa a moderada, os estudos sustentam a existência de uma relação positiva entre a prática de atividade física na adolescência e a consequente realização na fase adulta, porém o quanto seria necessário para que o indivíduo permanecesse ativo ao longo da vida não está claro (HALLAL et al., 2006), assim como o tipo de atividade física específica para produzir maiores chances de prática na fase adulta (TAMMELIN et al., 2003).

\section{Conclusões}

Constatou-se que 23,1\% dos acadêmicos do curso de Licenciatura em Educação Física da UESC foram considerados inativos no lazer. As mulheres apresentaram maior frequência à inatividade física atual que os homens e, de maneira análoga, a frequência em relação aos demais indicadores sóciodemográficos foram superiores para as mulheres. Destaca-se que a demanda de atividades curriculares, assim como a possibilidade de inserção no mercado de trabalho, podem contribuir para uma prática de atividade física no lazer em níveis considerados não desejáveis, conforme observado pela frequência superior de inatividade física em acadêmicos que cursavam os últimos períodos de universidade (2004).

Em relação à associação entre os indicadores de atividade física pregressa (escolar e não escolar) e a atividade física no lazer atual, observou-se diferença estatística para o contexto não escolar, que englobou a participação em atividades estruturadas, como os jogos em equipes e/ou clubes (escolinhas) esportivos, danças e lutas, em forma de 
competição e/ou apresentação. As análises demonstraram frequência de inatividade física no lazer de 42,1\% em acadêmicos de Educação Física inativos fisicamente em contexto não escolar, em função do gênero estas observações foram identificadas para as mulheres.

As informações sobre a prática de atividade física regular na adolescência demonstraram serem indicadores de atividades que podem contribuir para a prática de atividade física na vida adulta. Como os hábitos de atividade física, obtidos durante a infância e a adolescência, convergem durante a vida inteira, parece-nos importante que as atividades esportivas sejam estimuladas e desenvolvidas nesses grupos etários mais jovens. Em contrapartida, vale destacar o importante papel do profissional de Educação Física no estímulo à prática desde as idades iniciais, e consequente realização ao longo da vida. De outro lado, sugere-se que estudos com desenho longitudinal sejam realizados, visando caracterizar a relevância da prática de atividade física em âmbito escolar para um estilo de vida ativo na vida adulta.

\title{
Agradecimentos
}

Ao Grupo de Pesquisa em Atividade Física e Saúde (GPAF), pelo suporte na fase de coleta, Prof. Erick Fróes Almeida, Profa. Viviane Valentim Alves, Prof. Leandro Garcia Doroteio, Prof. Msc. Silvio Aparecido Fonseca e aos acadêmicos do curso de Educação Física que participaram do estudo PAFIS - UESC/BAHIA.

\section{Association between indicators physical activities practices in adolescence with current level of leisure-time physical activities practices in students of course of Physical Education in northeast of Brazil}

\begin{abstract}
The purpose was identified the frequency of leisure-time inactive current and association with indicators of physical activity in adolescence, context school and no, in students of course Physical Education. It was carried out cross-sectional study in 105 students of course. The frequency of leisure-time inactive current was $23.1 \%$ and identified associations for gender $(\mathrm{p}=0.003)$, with higher values for woman (38.6\%). Statistical associations were found in inactive students today when they were asked about the practice in adolescents in non-school context ( $\mathrm{p}=0.047)$. The Physical Education Teacher should encourage the practice of physical activities since the early ages, contributing for practice of lifelong.
\end{abstract}

Keyworks: Leisure; Adolescent; Students

\section{Académicos del curso de Educación Física en el nordeste de Brasil}

\footnotetext{
Resumen

El objetivo fue identificar la frecuencia de inactivos en recreo actual y la asociación con la práctica de la actividad física en la adolescencia en el contexto escolar y no escolar en académicos del curso de Educación Física. Fue realizado un estudio transversal en que participaran 105 estudiantes del curso. La frecuencia de académicos inactivos en el recreo fue $23,1 \%$ con diferencia significativa entre los sexos $(p=0,003)$, siendo mayor la frecuencia entre las mujeres (38,6\%). Asociaciones fueran evidenciadas en académicos inactivos actual y la práctica de la actividad física realizada en el contexto no escolar ( $\mathrm{p}=0,047)$. El profesor de Educación Física debe fomentar la práctica de actividades físicas buscando el contento por la práctica en toda la vida.
}

Palabras clave: Recreo - Adolescente - Estudiantes. 


\section{Referências}

ABOLFOTOUH, M. A. et al. Health-related lifestyle and risk bahaviours among students living in Alexandria University hostels. East Mediterr Health J., Egypt, v. 13, n. 2, p. 376-91, mar./abr., 2007.

ALVES, J. G. B. et al. Prática de esportes durante a adolescência e atividade física no lazer na vida adulta. Rev Bras Med Esporte, Niterói, v. 11, n. 5, p. 291-94, set./out., 2005.

BARROS, M. V. G. Atividades físicas no lazer e outros comportamentos relacionados à saúde dos trabalhadores da indústria no estado de Santa Catarina, Brasil. 1999. 131f. Dissertação (Mestrado em Educação Física) - Curso de Educação Física, Centro de Desportos, Universidade Federal de Santa Catarina, Florianópolis, 1999.

BARROS, M. V. G.; NAHAS, M. V. Comportamento de risco, auto-avaliação do nível de saúde e percepção de estresse entre trabalhadores da indústria. Rev Saúde Publ, São Paulo, v. 35, n. 6, p. 554-63, dez., 2001.

BIELEMANN, R. et al. Prática de atividade física no lazer entre acadêmicos de Educação Física e fatores associados. Revista Brasileira de Atividade Física \& Saúde, Florianópolis, v. 12, n. 3, p. 65-72, set./dez., 2007.

BRASIL. Ministério da Saúde. Instituto Nacional do Câncer. Coordenação de Prevenção e Vigilância. Inquérito domiciliar sobre comportamentos de risco e morbidade referida de doenças e agravos não-transmissíveis: Brasil. 15 capitais e Distrito Federal, 2002-2003. 183f. Rio de Janeiro: INCA. 2004.

CONROY, M. B. et al. Past physical activity, current physical activity, and risk of coronary heart disease. Med Sci Sports Exerc, USA, v. 37, n. 8, p. 1251-56, ago., 2005.

FRANCA, C.; COLARES, V. Estudo comparativo de condutas de saúde entre universitários no início e no final do curso. Rev Saude Publ, São Paulo, v. 42, n. 3, p. 420-7, 2008.

GIROTTO, C. A. et al. Prevalencia de factores de riesgo cardiovascular en ingresantes universitarios. Rev Saude Publ, São Paulo, v. 30, n. 6, p. 576-86, dez.,1996.

GORDON-LARSEN, P. et al. Longitudinal physical activity and sedentary behavior Trends. Adolescence to Adulthood. Am J Prev Med, Netherlands, v. 27, n. 4, p. 277-83, out., 2004.

GUEDES, D. P.; SANTOS, C. A.; LOPES, C. C. Estágios de mudança de comportamento e prática habitual de atividade física em universitários. Rev Bras Cineantropom Desempenho Hum, Florianópolis, v. 8, n. 4, p. 5-15, out./dez., 2006.

HALLAL, P. C. et al. Physical inactivity: prevalence and associated variables in brazilian adults. Med Sci Sports Exerc, USA, v. 35, n. 11, p. 1894-900, nov., 2003.

HALLAL, P. C. et al. Adolescent physical activity and health. A systematic review. Sports Med, New Zeland, v. 36, n.12, p. 1019-30, dez., 2006. 
MARCONDELLI, P. et al. Nível de atividade física e hábitos alimentares de universitários do $3^{\circ}$ ao $5^{\circ}$ semestres da área de saúde. Rev Nutr, Campinas, v. 21, n. 1, p. 39-47, jan./fev., 2008.

MOURA, E.C. et al. Vigilância de fatores de risco para doenças crônicas por inquérito telefônico nas capitais dos 26 estados brasileiros e no Distrito Federal (2006). Rev Bras Epidemiol, São Paulo, v. 11, sup. 1, p. 20-37, mai., 2008.

OLIVEIRA, M. et al. Estudos de crenças e expectativas acerca do álcool em estudantes universitários. Int J Clin Health Psychol, Granada, v. 7, n. 2, p. 421-33, mai., 2007.

PAFFENBARGER et al. Physical activity, all-cause mortality, and longevity of college alumni. N Eng1 J Med, USA, v. 314, n. 10, p. 605-13, mar., 1986.

PAFFENBARGER et al. The association of changes in physical-activity level and other lifestyle characteristics with mortality among men. N EnglJ Med, USA, v. 328, n. 8, p. 538-45, fev., 1993.

PITANGA, F. J. G; LESSA, I. Associação entre inatividade física no tempo livre e internações hospitalares em adultos da cidade de Salvador - Brasil. Rev Bras Cineantropom Desempenho Hum, Florianópolis, v. 10, n. 4, p. 347-353, out./dez., 2008.

RABELO, L. M. et al. Risk factors for atherosclerosis in students of a private university in São Paulo - Brazil. Arq Bras Cardiol, Rio de Janeiro, v. 72, n. 5, p. 575-80, mai., 1999.

RODRIGUES, E. S. R. Nível de atividade física e tabagismo em universitários. Rev Saude Publ, São Paulo, v. 42, n. 4, p. 672-8, ago., 2008.

SILVA, G. S. F. et al. Avaliação do nível de atividade física de estudantes de graduação das áreas saúde/biologia. Rev Bras Med Esporte, Niterói, v. 13, n. 1, p. 39-42, jan./fev., 2007.

SOUSA, T. F. Autoavaliação do nível de saúde em estudantes de Educação Física. Revista Saúde e Pesquisa, Maringá, v. 2, n. 1, p. 17-21, jan./abr., 2009.

STOCK, C. et al. Gender-specific health behaviors of German university students predict the interest in campus health promotion. Health Promot Int, England, v. 16, n. 2, p. 145-54, jun., 2001.

TAMMELIN, T. et al. Adolescent participation in sports and adult physical activity. Am J Prev Med, Netherlands, v. 24, n. 1, p. 22-28, jan., 2003.

TELAMA, R. et al. Physical activity from childhood to adulthood A 21-year tracking study. Am J Prev Med, Netherlands, v. 28, n. 3, p. 267-73, abr., 2005.

UNITED STATES DEPARTMENT OF HEALTH AND HUMAN SERVICES (USDHHS). Physical activity and health: a report of the Surgeon General. Atlanta, U.S. Departament of Health and Human Services, Centers for Disease and Prevention, National Center for Chronic Disease Prevention and Health Promotion, 
1996. Disponível em: < http://www.cdc.gov/nccdphp/sgr/contents.htm> . Acesso em: 13 jan. 2006.

UNITED STATES DEPARTMENT OF HEALTH AND HUMAN SERVICES (USDHHS). Physical Activity Guidelines for Americans. Washington, 2008. Disponível em: <http://www.health.gov/paguidelines/pdf/paguide.pdf >. Acesso em: 20 out. 2008.

WORLD HEALTH ORGANIZATION (WHO). The world health report 2002. Reducing risks, promoting healthy life. Geneva, 2002. Disponível em: <http://www. who.int/whr/2002/en/whr02_en.pdf>. Acesso em: 24 jan. 2008.

YUSUF, S. et al. Effect of potentially modifiable risk factors associated with myocardial infarction in 52 countries (the INTERHEART study): case-control study. Lancet, England, v. 364, p. 937-52, set., 2004.

Recebido em: 03/07/2009

Revisado em:11/08/2009

Aprovado em: 08/09/2009

\section{Endereço para contato}

Thiago Ferreira de Sousa

Universidade Federal de Santa Catarina

Coordenadoria de Pós-Graduação em Educação Física

Campus Universitário,

CEP: 88.040-900

Florianópolis - Santa Catarina - Brasil

E-mail:tfsousa_thiago@yahoo.com.br 